International Journal of Pure and Applied Mathematics

Volume 106 No. 4 2016, 1003-1016

ISSN: 1311-8080 (printed version); ISSN: 1314-3395 (on-line version)

url: http://www.ijpam.eu

doi: 10.12732 /ijpam.v106i4.3

\title{
ANALYTIC SOLUTIONS FOR NONLINEAR EVOLUTION EQUATIONS IN MATHEMATICAL PHYSICS
}

\author{
Khaled A. Gepreel ${ }^{1,2} \S$, Taher A. Nofal ${ }^{1,3}$ \\ ${ }^{1}$ Department of Mathematics \\ Faculty of Science \\ Taif University \\ KINGDOM SAUDI ARABIA \\ ${ }^{2}$ Department of Mathematics \\ Faculty of Science \\ City Zagazig University \\ Zagazig, EGYPT \\ ${ }^{3}$ Department of Mathematics \\ Faculty of Science \\ El-Minia University \\ EGYPT
}

\begin{abstract}
In this article, we use the modified $(w / g)$ - expansionmethod to find the traveling wave solutions for some nonlinear partial differential equations in mathematical physicsvia the nonlinear variant Boussinesq differential equations. When $w$ and $g$ are taken special choices, some families of direct expansion methods are obtained to obtain the exact solutions for the nonlinear evolutions equations in the mathematical physics. Based on these interesting results, we further give two forms of expansions via the modified $g^{\prime} / g^{2}$ - expansion method and modified $g^{\prime}$ - expansion methods. When the parameters are taken some special values, the solitary wave are derived from the traveling waves. This method is reliable, simple, and gives many new exact solutions.
\end{abstract}

AMS Subject Classification: $35 \mathrm{~K} 99,35 \mathrm{P} 05,35 \mathrm{P} 99$

Key Words: the modified $(w / g)$-expansion method, traveling wave solutions, the nonlinear variant Boussinesq differential equations

Received: October 29, 2015

Published: March 6, 2016

$\S$ Correspondence author (c) 2016 Academic Publications, Ltd. url: www.acadpubl.eu 


\section{Introduction}

Nonlinear partial differential equations play an important role in describing the various phenomena not only in physics, but also in biology and chemistry, and several other fields of science and engineering. It is one of the important jobs in the study of the nonlinear partial differential equations are searching for finding the traveling wave solutions. There are many methods for obtaining the exact solutions to nonlinear partial differential equations such as the inverse scattering method [1], Hirota's bilinear method [2],Backlund transformation [3], the first integral method [4], Painlev expansion [5], sine-cosine method [6], homogenous balance method [7], extended trial equation method [8,9], perturbation method [10,11], variation method [12], tanh- function method [13,14],Jacobi elliptic function expansion method $[15,16]$, Exp-function method $[17,18]$ and Fexpansion method [19,20]. Wang et al [21] suggested a direct method called the $\left(G^{\prime} / G\right)$ - expansion method to find the rational traveling wave solutions for nonlinear partial differential equations (NPDEs).Recently, Li et al [22] presented the modified $(w / g)$ - expansion method to find the traveling wave solutions for the Vakhnenko equation. Also Zayed et al [23] used the modified $(w / g)$ - expansion method to solve the modified generalized Vakhnenko equation. In this paper, we use the modified $(w / g)$ - expansion method, where $w, g$ are arbitrary function to find the exact solutions to nonlinear evolution equations in mathematical physics. We obtain some new kind of traveling wave solutions when the parameter is taking some special values.

\section{Description of the Modified $(w / g)$-Expansion Method for NPDEs}

In this section,we illustrate the mean idea of the modified $(w / g)$ - expansion method which discussed in $[22,23]$.For a given nonlinear partial differential equation

$$
P\left(u, u_{t}, u_{x}, u_{t t}, u_{x x}, u_{x t}, . .\right)=0,
$$

where $u=u(x, t)$ is an unknown function, $P$ is a polynomial in $u=u(x, t)$ and its various partial derivatives, in which the highest order derivatives and nonlinear terms are involved.

Step 1. We use the travelling wave transformation:

$$
u=U(\xi), \quad \xi=x-k t,
$$

where $k$ is a nonzero constant.Substituting Eq.(2) into Eq.(1), we recover the ordinary differential equation (ODE) 


$$
Q\left(U, U^{\prime}, U^{\prime \prime}, \ldots\right)=0
$$

Step 2.Suppose the solution of Eq.(3) can be expressed by a polynomial in a finite form of $(w / g)$ as following:

$$
U(\xi)=\sum_{i=0}^{m} a_{i}\left(\frac{w(\xi)}{g(\xi)}\right)^{i}
$$

where $a_{i}(i=0,1, \ldots, m)$ are arbitrary constants to be determined later , and $w(\xi), g(\xi)$ satisfy the following relation

$$
\left(\frac{w(\xi)}{g(\xi)}\right)^{\prime}=a+b\left(\frac{w(\xi)}{g(\xi)}\right)+c\left(\frac{w(\xi)}{g(\xi)}\right)^{2}
$$

or

$$
w^{\prime} g-w g^{\prime}=a g^{2}+b w g+c w^{2}
$$

where $a, b, c$ are arbitraryconstants.

Step 3. Determine the positive integer $\min$ the formula (4) by balancing the nonlinear term(s) and the highest order derivative in Eq. (3).

Step 4. Substituting Eq. (4) into (3) along with (5), and collect all terms with the same powers $(w / g)^{i},(i=0,1, \ldots, m)$. Setting each coefficient of $(w / g)^{i},(i=0,1, \ldots, m)$ to be zero, yield a set of algebraic equations for $a_{i}(i=0, \pm 1, \ldots, \pm m)$ and $k$.

Step 5. Solving these over-determined system of algebraic equations with the help of Maple software package to determine $a_{i}(i=0, \pm 1, \ldots, \pm m)$ and $k$.

Step 6. Using the results which obtained in the above steps to derives a series of fundamental solutions of nonlinear partial differential equation (1).

Remark 1. Furthermore if we put $g=1, b=0$, and $c=1$. In this case the solution (4) takes the form

$$
U(\xi)=\sum_{i=0}^{m} a_{i} w^{i}
$$

where $a_{i}(i=0,1, \ldots, m)$ are arbitrary constants, and $w$ satisfy the following relation

$$
w^{\prime}=a+w^{2}
$$


In this case the $(w / g)$ - expansion method is equivalent to the tanh- function method.Also when $g=1$, and $a, b, c$ are nonzero constants the $(w / g)$ - expansion method is equivalent to the Riccati expansion function method $[13,14]$.

Remark 2. (see $[22,23]$ ) If we choose $w=g^{\prime}, a=-\mu, b=-\lambda$, and $c=-1$. In this case the solution (4) takes the form

$$
U(\xi)=\sum_{i=0}^{m} a_{i}\left(\frac{g^{\prime}}{g}\right)^{i},
$$

where $a_{i}(i=0,1, \ldots, m)$ are arbitrary constants, and $g$ satisfy the following second order differential equation:-

$$
g^{\prime \prime}+\lambda g^{\prime}+\mu g=0
$$

In this case the $(w / g)$ - expansion method is equivalent to the $\left(G^{\prime} / G\right)$ expansion method proposed by Wang et al, see [21].

Remark 3. If we put $w=g^{\prime} / g, \quad b=0$, . We have a new form of exact solution takes the form

$$
U(\xi)=\sum_{i=0}^{m} a_{i}\left(\frac{g^{\prime}}{g^{2}}\right)^{i},
$$

where $a_{i}(i=0,1, \ldots, m)$ are arbitrary constants, and $g$ satisfy the following nonlinear second order differential equation

$$
g^{2} g^{\prime \prime}-2 g\left(g^{\prime}\right)^{2}=a g^{4}+c\left(g^{\prime}\right)^{2},
$$

which is called $\left(g^{\prime} / g^{2}\right)$ - expansion method and proposed in [22,23].

Remark 4. If we put $w=g g^{\prime}$. We have a new form of exact solution takes the form

$$
U(\xi)=\sum_{i=0}^{m} a_{i}\left(g^{\prime}\right)^{i},
$$

where $a_{i}(i=0,1, \ldots, m)$ are arbitrary constants, and $g$ satisfy the nonlinear second order differential equation

$$
g^{\prime \prime}=a+b g^{\prime}+c\left(g^{\prime}\right)^{2},
$$

which is called $\left(g^{\prime}\right)$-expansion method and proposed in $[22,23]$. 


\section{Applications of Extended Rational $(w / g)$ Expansion Method for NPDEs}

In this section, we use the two expansions methods namely the $\left(g^{\prime} / g^{2}\right)$ expansion method and $\left(g^{\prime}\right)$-expansion method tofind the traveling wave solutions for nonlinear evolution equations in mathematical physics via the nonlinear variant Boussinesq differential equations which are very important in the mathematical science and have been paid attention by many researchers in physics and engineering.

\subsection{Traveling Wave Solutions of the Nonlinear Variant Boussinesq Differential Equations}

The Boussinesq equation is a well-known model of long water wave of moderate amplitude, describes one dimensional, weakly nonlinear internal wave which develops at the boundary between two immiscible fluids. Guo et al (see [24]) model of the atmospheric movement equation which is applicable to mesoscale and quashing compressible fluid movement, which means important physical applications in hydrodynamics. The Boussinesq equation also is of considerable mathematic interests because of its rich mathematical structures. In the present research, we focus on the variant Boussinesq equations, which was derived by Sachs (see [25]) in 1988 as a model for water waves:

$$
\begin{aligned}
& H_{t}+(H u)_{x}+u_{x x x}=0 \\
& u_{t}+H_{x}+u u_{x}=0 .
\end{aligned}
$$

where $u(x, t)$ is the velocity, $H(x, t)$ is the height of free wave surface for fluid in the trough, and the subscripts denote partial derivatives. In the past years, many authors have studied Eqs. (15). For example, Wang [26] solved Eq. (15) by the homogeneous balance method. Yan etal [27] obtained new explicit and exact traveling wave solutions for Eq. (15) by an improved sinecosine method and the Wu elimination method. Naz et al. [28] obtained the conservation laws for Eq. (15) by an interesting method of increasing the order of partial differential equations. Fan etal [29] uniformly constructed a series of traveling wave solutions for Eq. (15) by a new algebraic method. Lü [30] solved Eq. (15) by a general Jacobi elliptic function expansion method, and obtained Jacobi elliptic function solutions. Yuan et al. [31] constructed bifurcations of traveling wave solutions for Eq. (15) by the bifurcation theory of planar dynamical systems. Li et al. [32] obtained all possible smooth, cusped solitary wave solutions for Eq. (15) by the phase portrait analytical technique.Let us 
assume the traveling wave solutions of Eq. (15) in the following form:

$$
u(x, t)=U(\xi), \quad H(x, t)=H(\xi), \xi=x-k t
$$

where $k$ is an arbitrary constant. The transformation (16) permits us to convert PDE's (15) to the following ODE's :-

$$
\begin{aligned}
& -k H+H U+U^{\prime \prime}+C_{1}=0, \\
& -K U+H+\frac{1}{2} U^{2}+C_{2}=0 .
\end{aligned}
$$

where $C_{1}$ and $C_{2}$ are the integration constants.

\subsubsection{The Modified $\left(g^{\prime} / g^{2}\right)$-Expansion Method for Nonlinear Variant Boussinesq Differential Equations}

In this subsection we use the direct method $\left(g^{\prime} / g^{2}\right)$ - expansion method to find the traveling wave solutions of Eqs. (17). By balancing the highest order derivative term and nonlinear terms in Eq. (17), we suppose the solutions of Eqs. (17) have the following form:

$$
\begin{aligned}
& U(\xi)=a_{0}+a_{1}\left(\frac{g^{\prime}}{g^{2}}\right), \\
& H(\xi)=b_{0}+b_{1}\left(\frac{g^{\prime}}{g^{2}}\right)+b_{2}\left(\frac{g^{\prime}}{g^{2}}\right)^{2},
\end{aligned}
$$

where $a_{0}, a_{1}, b_{0}, b_{1}$ and $b_{2}$ are constants to be determined later and $g(\xi)$ satisfies equation (12). Substituting Eq. (18) along with (12) into Eq. (17) and collecting all terms with the same order of $\left(g^{\prime} / g^{2}\right)$ together, the left hand side of Eq. (17) are converted into polynomial in $\left(g^{\prime} / g^{2}\right)$. Setting each coefficient of these polynomials to be zero, we derive a set of algebraic equations for $a_{0}, a_{1}, b_{0}, b_{1}, b_{2}$ and $k$. Solving the set of algebraic equations by using Maple or Mathematica, software package to get the following results:

$$
\begin{aligned}
& C_{2}=\frac{1}{2} k^{2}+2 c a, \quad a_{0}=k, \quad, a_{1}=-2 c, \quad b_{0}=-2 c a, \quad b_{2}=-2 c^{2}, \\
& b_{1}=C_{1}=0
\end{aligned}
$$

where $k, c, a$ are arbitrary constants. The general solution of the nonlinear differential equation (15) have the following forms:

Family 1. If $c a>0$,

$$
g(\xi)=\frac{2 c}{\ln \left(\frac{c}{a}\left(A_{1} \sin (\sqrt{a c} \xi)-A_{2} \cos (\sqrt{a c} \xi)\right)^{2}\right)}
$$


and

$$
\frac{g^{\prime}}{g^{2}}=\sqrt{\frac{a}{c}} \frac{A_{1} \cos (\sqrt{a c} \xi)+A_{2} \sin (\sqrt{a c} \xi)}{A_{1} \sin (\sqrt{a c} \xi)-A_{2} \cos (\sqrt{a c} \xi)}
$$

In this family the traveling wave solution of (15) takes the following form:

$$
\begin{aligned}
& U(\xi)=k-2 \sqrt{a c}\left(\frac{A_{1} \cos (\sqrt{a c} \xi)+A_{2} \sin (\sqrt{a c} \xi)}{A_{1} \sin (\sqrt{a c} \xi)-A_{2} \cos (\sqrt{a c} \xi)}\right), \\
& H(\xi)=-2 c a-2 c a\left(\frac{A_{1} \cos (\sqrt{a c} \xi)+A_{2} \sin (\sqrt{a c} \xi)}{A_{1} \sin (\sqrt{a c} \xi)-A_{2} \cos (\sqrt{a c} \xi)}\right)^{2} .
\end{aligned}
$$

where $\xi=x-k t$.

Family 2. If $c a<0$,

$$
g(\xi)=-\frac{2 c}{2 \sqrt{|a c|} \xi-\ln \left[\frac{c}{4 a}\left(A_{1} e^{2 \sqrt{|a c|} \xi}-A_{2}\right)^{2}\right]}
$$

and

$$
\frac{g^{\prime}}{g^{2}}=\frac{1}{2 c}\left[2 \sqrt{|a c|}-\frac{4 \sqrt{|a c|} A_{1} e^{2 \sqrt{|a c|} \xi}}{A_{1} e^{2 \sqrt{|a c|} \xi}-A_{2}}\right]
$$

In this family the traveling wave solutions of (15) take the following form:

$$
\begin{aligned}
& U(\xi)=k-\left(2 \sqrt{|a c|}-\frac{4 \sqrt{|a c|} A_{1} e^{2 \sqrt{|a c|} \xi}}{A_{1} e^{2 \sqrt{|a c|} \xi}-A_{2}}\right), \\
& H(\xi)=-2 c a-\frac{1}{2}\left(2 \sqrt{|a c|}-\frac{4 \sqrt{|a c|} A_{1} e^{2 \sqrt{|a c|} \xi}}{A_{1} e^{2 \sqrt{|a c|} \xi}-A_{2}}\right)^{2},
\end{aligned}
$$

Family 3. If $a=0, \quad c \neq 0$,

$$
g(\xi)=\frac{c}{\ln \left(A_{1} c \xi+A_{2} c\right)}
$$

and

$$
\frac{g^{\prime}}{g^{2}}=-\frac{A_{1}}{A_{1} c \xi+A_{2} c}
$$

In this family the traveling wave solutions of (15) take the following form:

$$
\begin{aligned}
& U(\xi)=k+\frac{2 A_{1} c}{A_{1} c \xi+A_{2} c}, \\
& H(\xi)=-2 c a-\frac{2 c^{2} A_{1}^{2}}{\left(A_{1} c \xi+A_{2} c\right)^{2}} .
\end{aligned}
$$




\subsubsection{The New Modified $g^{\prime}$-Expansion Method for Nonlinear Variant Boussinesq Differential Equations}

In this subsection we use the direct method $\left(g^{\prime} / g^{2}\right)$ - expansion method to find the traveling wave solutions of Eqs. (17). By balancing the highest order derivative term and nonlinear terms in Eq. (17), we suppose the solutions of Eqs. (17) have the following form:

$$
\begin{aligned}
& U(\xi)=a_{0}+a_{1} g^{\prime}, \\
& H(\xi)=b_{0}+b_{1} g^{\prime}+b_{2} g^{2},
\end{aligned}
$$

where $a_{0}, a_{1}, b_{0}, b_{1}$ and $b_{2}$ are constants to be determined later and $g(\xi)$ satisfies equation (14). Substituting Eq. (29) along with (14) into Eq. (17) and collecting all terms with the same order of $g^{\prime}$ together, the left hand side of Eq. (17) are converted into polynomial in $g^{\prime}$. Setting each coefficient of these polynomials to be zero, we derive a set of algebraic equations for $a_{0}, a_{1}, b_{0}, b_{1}$, $b_{2}$ and $k$. Solving the set of algebraic equations by using Maple or Mathematica , software package to get the following results:

$$
\begin{array}{cc}
C_{2}=\frac{1}{2} k^{2}-\frac{1}{2} b^{2}+2 c a, & a_{0}=k-b, \\
b_{1}=-2 b c, \quad b_{2}=-2 c^{2}, & C_{1}=0
\end{array}
$$

where $k, c, a$ are arbitrary constants. The traveling wave solution of the nonlinear differential equation (17) have the following forms:

Family 1. If $\Delta=4 a c-b^{2}>0$,

$$
g(\xi)=\frac{1}{2 c}\left[\ln \left(1+\tan ^{2}\left(\frac{1}{2} \sqrt{\Delta} \xi\right)\right)-b \xi\right]
$$

and

$$
g^{\prime}=\frac{1}{2 c}\left[\sqrt{\Delta} \tan ^{2}\left(\frac{1}{2} \sqrt{\Delta} \xi\right)-b\right] .
$$

In this family the traveling wave solutions of (15) take the following form:

$$
\begin{aligned}
& U(\xi)=k-b-\left[\sqrt{\Delta} \tan ^{2}\left(\frac{1}{2} \sqrt{\Delta} \xi\right)-b\right] \\
& H(\xi)=-2 c a-b\left[\sqrt{\Delta} \tan ^{2}\left(\frac{1}{2} \sqrt{\Delta} \xi\right)-b\right]-\frac{1}{2}\left[\sqrt{\Delta} \tan ^{2}\left(\frac{1}{2} \sqrt{\Delta} \xi\right)-b\right]^{2}
\end{aligned}
$$

Family 2. If $\Delta=4 a c-b^{2}<0$, 


$$
g(\xi)=\frac{1}{2 c}\left[\ln \left(\tanh ^{2}\left(\frac{1}{2} \sqrt{-\Delta} \xi\right)-1\right)-b \xi\right]
$$

and

$$
g^{\prime}=\frac{1}{2 c}\left[\sqrt{-\Delta} \tanh ^{2}\left(-\frac{1}{2} \sqrt{-\Delta} \xi\right)-b\right] .
$$

In this family the traveling wave solutions of (15) take the following form:

$$
\begin{aligned}
U(\xi) & =k-b-\left[\sqrt{-\Delta} \tanh ^{2}\left(-\frac{1}{2} \sqrt{-\Delta} \xi\right)-b\right] \\
H(\xi) & =-2 c a-b\left[\sqrt{-\Delta} \tanh ^{2}\left(-\frac{1}{2} \sqrt{-\Delta} \xi\right)-b\right] \\
& -\frac{1}{2}\left[\sqrt{-\Delta} \tanh ^{2}\left(-\frac{1}{2} \sqrt{-\Delta} \xi\right)-b\right]^{2}
\end{aligned}
$$

Family 3. If $\Delta=4 a c-b^{2}=0$,

$$
g(\xi)=-\frac{1}{c}\left[\ln (\xi)+\frac{b}{2} \xi\right]
$$

and

$$
g^{\prime}=-\frac{1}{c}\left(\frac{1}{\xi}+\frac{b}{2} .\right) .
$$

In this family the traveling wave solutions of (15) take the following form:

$$
\begin{aligned}
& U(\xi)=k-b+2\left(\frac{1}{\xi}+\frac{b}{2}\right) \\
& H(\xi)=-2 c a+2 b\left(\frac{1}{\xi}+\frac{b}{2}\right)-2\left(\frac{1}{\xi}+\frac{b}{2}\right)^{2}
\end{aligned}
$$

\subsubsection{Numerical Solutions for the Exact Solutions for Nonlinear Variant Boussinesq Differential Equations}

In this section we give some figures to illustrate the behavior of the exact solutions which obtained in above sections To this end, we select some special values of the parameters to show the behavior of extended rational $\left(g^{\prime} / g^{2}\right)$ expansion method and $g^{\prime}$ expansion method for nonlinear variant Boussinesq differential equations 

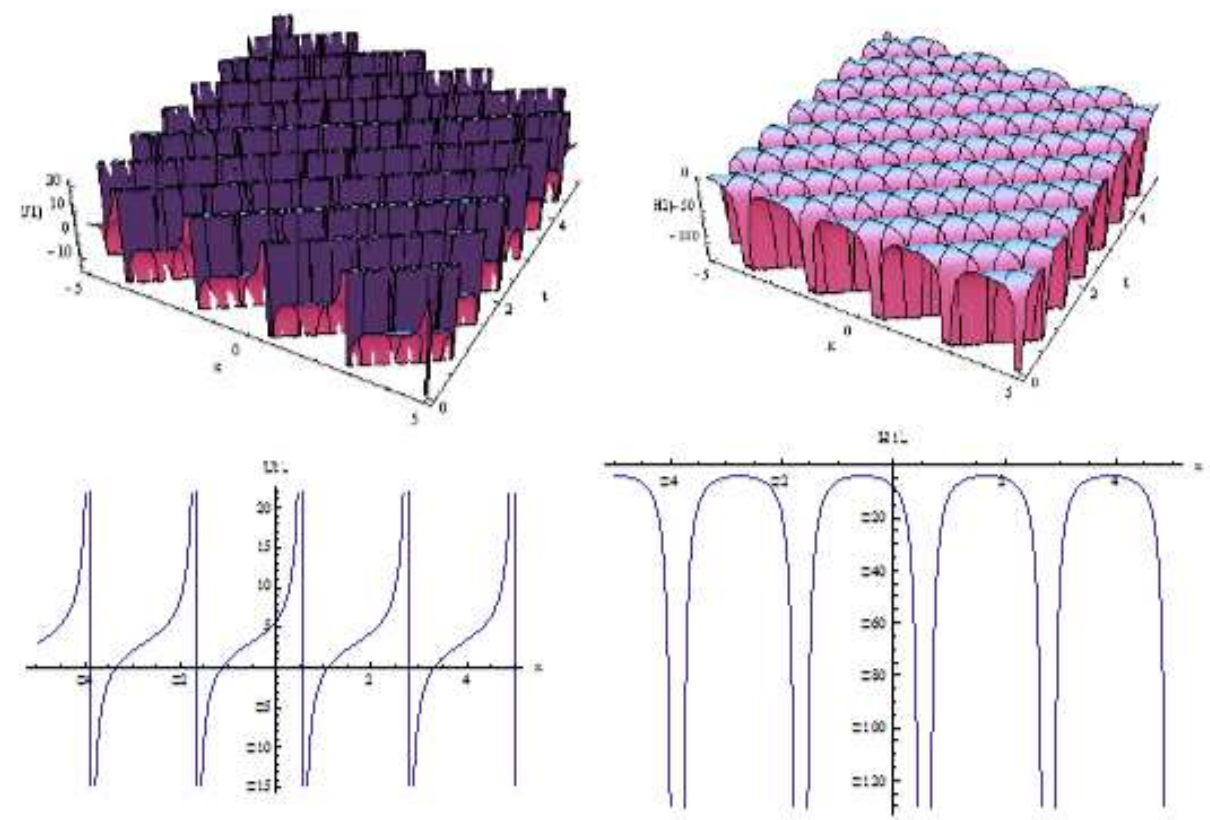

Figure 1: The exact solution (22) of Eq. (16) and its projection at $t=0$ when $k=3, a=2, c=1, A_{1}=5, A_{2}=3$

\section{Conclusion}

In this paper we used the rational direct method $(w / g)$ expansion method to construct a series of some new traveling wave solutions for some nonlinear partial differential equations in the mathematical physics. We constructed the rational exact solutions in many different functions such as hyperbolic function solutions, trigonometric function solutions and rational exact solution. The performance of this method reliable, effective and powerful for solving the nonlinear partial differential equations. Also we illustrate the behavior of the traveling wave solutions when the parameters take some special values. 



Figure 2: The exact solution (25) of Eq. (15) and its projection at $t=0$ when $k=3, a=2, c=1, A_{1}=5, A_{2}=3$ 

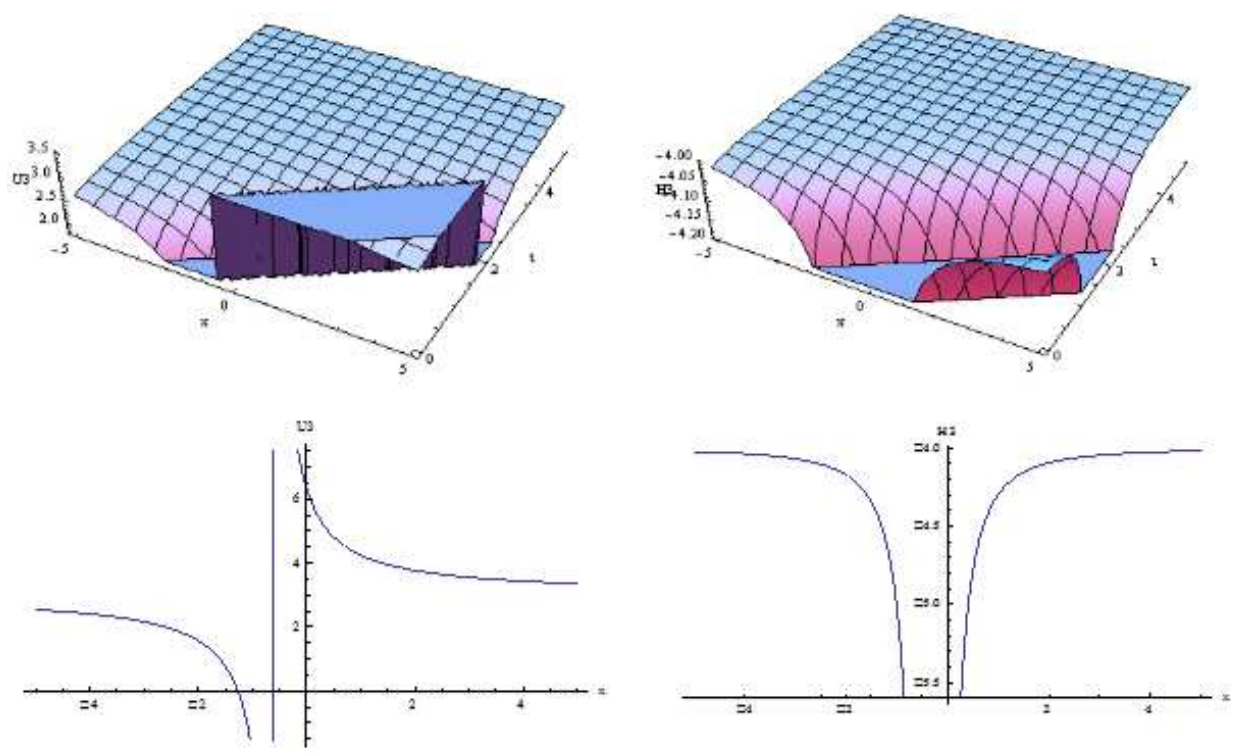

Figure 3: The exact solution (28) of Eq. (15) and its projection at $t=0$ when $k=3, a=2, c=1, A_{1}=5, A_{2}=3$ 


\section{References}

[1] M.J. Ablowitz and P.A. Clarkson, Solitons, nonlinear Evolution Equations and Inverse Scattering Transform, Cambridge Univ. Press, Cambridge, (1991).

[2] R.Hirota, Exact solution of the KdV equation for multiple collisions of solutions, Phys. Rev. Letters, 27 (1971) 1192-1194.

[3] M.R.Miura, Backlund Transformation,Springer-Verlag, Berlin,1978.

[4] A. Bekir , F. Tascan and O. Unsal, Exact solutions of the Zoomeron and Klein Gordon Zahkharov equations, J. Associat. Arab Univ. Basic Appl. Sci., 17 (2015) 1-5.

[5] J.Weiss, M.Tabor and G.Garnevalle, The Paineleve property for partial differential equations, J.Math.Phys. 24 (1983) 522-526

[6] D.S.Wang, Y.J.Ren and H.Q.Zhang, Further extended sinh-cosh and sin-cos methods and new non traveling wave solutions of the $(2+1)$-dimensional dispersive long wave equations, Appl. Math.E-Notes, 5 (2005) 157-163.

[7] M.L. Wang, Exact solutions for a compound KdV-Burgers equation , Phys. Lett. A, 213 (1996) 279-287.

[8] K.A. Gepreel and T.A. Nofal, Extended trial equation method for nonlinear partial differential equations, Z. Naturforsch A, 70 (2015) 269-279.

[9] F. Belgacem, H Bulut, H. Baskonus and T. Aktuk, Mathematical analysis of generalized Benjamin and Burger- KdV equation via extended trial equation method, J. Associat. Arab Univ. Basic Appl. Sci., 16 (2014) 91-100.

[10] J.H. He, Homotopy perturbation method for bifurcation of nonlinear wave equations, Int. J. Nonlinear Sci. Numer. Simul., 6 (2005) 207-208 .

[11] E.M.E.Zayed, T.A. Nofal and K.A.Gepreel, The homotopy perturbation method for solving nonlinear Burgers and new coupled MKdV equations, Zeitschrift fur Naturforschung, 63a (2008) $627-633$.

[12] H.M. Liu, Generalized variational principles for ion acoustic plasma waves by He's semiinverse method, Chaos, Solitons \& Fractals, 23 (2005) 573 -576.

[13] H.A.Abdusalam, On an improved complex tanh-function method, Int. J. Nonlinear Sci.Numer. Simul., 6 (2005) 99-106.

[14] E.M.E.Zayed ,Hassan A.Zedan and Khaled A. Gepreel, Group analysis and modifiedextended Tanh- function to find the invariant solutions and soliton solutions fornonlinear Euler equations, Int. J. Nonlinear Sci. Numer. Simul., 5 (2004) 221-234.

[15] Y.Chen and Q.Wang, Extended Jacobi elliptic function rational expansion method and abundant families of Jacobi elliptic functions solutions to $(1+1)$ dimensional dispersive long wave equation, Chaos, Solitons and Fractals, 24 (2005) 745-757.

[16] S.Liu, Z.Fu, S.D. Liu and Q.Zhao, Jacobi elliptic function expansion method and periodic wave solutions of nonlinear wave equations, Phys. Letters A, 289 (2001) 69-74.

[17] S. Zhang and T.C. Xia, A generalized F-expansion method and new exact solutions of Konopelchenko-Dubrovsky equations, Appl. Math. Comput., 183 (2006) 1190-1200 .

[18] J.H.He and X.H.Wu, Exp-function method for nonlinear wave equations, Chaos, Solitons and Fractals, 30 (2006) 700-708. 
[19] M.A. Abdou, The extended F-expansion method and its applications for a class of nonlinear evolution equation, Chaos, Solitons and Fractals, 31 (2007) 95 -104 .

[20] M.Wang and X.Li, Applications of F-expansion to periodic wave solutions for a new Hamiltonian amplitude equation, Chaos, Solitons and Fractals, 24 (2005) 1257- 1268.

[21] M.Wang, X.Li and J.Zhang, The $\left(G^{\prime} / G\right)$ - expansion method and traveling wave solutions of nonlinear evolution equations in mathematical physics, Phys.Letters A, 372 (2008) 417-423.

[22] W.A. Li, H. Chen and G. Zhang, The (w/g)-expansion method and itsapplication to Vakhnenko equation, Chinese Phys. B, 18 (2009), 400-409.

[23] E.M.E.Zayed and Ahmed H. Arnous, The modified (w/g)- expansion method and its applications for solving the modified generalized Vakhnenko equation, Italian Journal of Pure and Applied Mathematics, 32 (2014) 477-492.

[24] P. Guo, , X.Wu and L. Wang, Multiple soliton solutions for the variantBoussinesq equations, Advances in Difference Equations. 2015 (2015):37.

[25] R. Sachs, On the integrable variant of the Boussinesq system: Painlev property, rational solutions, a related many-body system, and equivalence with the AKNS hierarchy, Physica D 30 (1988) 1-27.

[26] M.Wang, Solitary wave solutions for variant Boussinesq equations, Phys. Lett. A, 199 (1995) 169-172.

[27] Z.Y.Yan and H. Zhang, New explicit and exact travelling wave solutions for a system of variant Boussinesq equations inmathematical physics, Phys. Lett. A, 252 (1999) 291-296

[28] R.Naz, F. Mahomed and T. Hayat, Conservation laws for third-order variant Boussinesq system. Appl. Math. Lett. 23 (2010) 883-886.

[29] E.Fan and Y. Hon, A series of travelling wave solutions for two variant Boussinesq equations in shallow water waves, Chaos Solitons Fractals, 15 (2003) 559-566.

[30] D. L, Jacobi elliptic function solutions for two variant Boussinesq equations, Chaos Solitons Fractals, 24 (2005) 1373-1385.

[31] Y.Yuan,D. Pu and S. Li, Bifurcations of travelling wave solutions in variant Boussinesq equations, Appl. Math. Mech. 27, (2006) 811-822 .

[32] H. Li, L. Ma and D. Feng, Single-peak solitary wave solutions for the variant Boussinesq equations, Pramana, 80 (2013) 933-944. 